\title{
Neuro-cognitive processes as mediators of psychological treatment effects
}

\author{
Andrea M.F. Reiter ${ }^{1,2,3,4}$, Nadim Atiya ${ }^{1,2}$, Isabel M. Berwian ${ }^{5}$ and Quentin J. M. Huys ${ }^{1,6,7}$
}

1 Max Planck UCL Centre for Computational Psychiatry and Ageing Research, University College London; 2 Wellcome Centre for Human Neuroimaging, Institute of Neurology, University College London, London 3 Center of Mental Health, Psychosomatics and Psychotherapy, University of Wuerzburg, Wuerzburg, Germany; 4 Chair of Lifespan Developmental Neuroscience, Faculty of Psychology, Technische Universität Dresden, Germany; 5 Princeton Neuroscience Institute, Princeton University, Princeton, USA; 6 Division of Psychiatry, University College London, London; 7 Camden and Islington NHS Foundation Trust

\section{Corresponding author}

Quentin Huys

Max Planck UCL Centre for Computational Psychiatry and Ageing Research

Russell Square House

10-12 Russell Square

WC1B 5EH London

United Kingdom

q.huys@ucl.ac.uk

\begin{abstract}
Psychological interventions are first-line treatments of depression. Despite a rich theoretical background, the mediators of treatment effects remain only partially understood: it has been difficult to precisely delineate the targets psychological interventions engage, and even more difficult to differentiate amongst the targets engaged by different psychological interventions. Here, we outline these issues and discuss a surprisingly understudied approach, namely the study of cognitive and computational tasks to measure psychological treatment targets. Such tasks benefit from substantial advances in cognitive neuroscience over the past two decades, and have excellent face validity. We discuss two candidate tasks for backtranslation and conclude with a critical evaluation of potential problems associated with this neuro-cognitive approach.
\end{abstract}

\section{Highlights}

- Cognitive Behavioural Therapy (CBT) for depression has a rich theoretical background in cognitive and learning theories, but we still know little about the specific mechanisms engaged by different CBT modules.

- Neuro-computational probes derived from modern computational cognitive neuroscience are specifically designed to characterise behaviours and cognitions that CBT aims to change

- Cognitive computational tasks might be useful tools for research aimed at defining mechanisms and mediators brought about by different CBT modules.

- We discuss metacognition as candidate mediators of Cognitive Restructuring, and Reward and Effort-based decision-making as candidate mediators for Behavioural Activation

- The psychometric properties of tasks pose obstacles for their clinical use. 


\section{Introduction}

Depression is amongst the most burdensome illnesses worldwide ${ }^{1}$. A large treatment gap exists, both due to insufficient provision of treatment and due to limited efficacy of existing treatments ${ }^{2}$. There is an urgent need for treatments that better engage the mechanisms causing the illness. Cognitive Behavioural Therapy (CBT), for instance, is a standard first-line treatment ${ }^{3}$ with proven efficacy established in numerous randomized controlled trials ${ }^{4}$. However, it has been surprisingly difficult to establish the mechanisms by which psychotherapeutic treatments such as CBT work, and even more difficult to differentiate the mechanisms engaged by different forms of therapy. The latter resulted in a prominent claim that different psychotherapies, regardless of their specific ingredients, lead to similar outcomes ("Dodo bird effect") 5 .

Part of this is likely to arise from the fact that different psychological interventions contain similar components, or component interventions ${ }^{5}$. Arguably, the lack of knowledge about which interventions affect which specific mechanisms is an important factor maintaining the treatment gap. First, it prevents precision treatment because it is not possible to measure the relevant mechanisms in order to allocate treatments individually. For instance, it prevents the optimization of existing treatment manuals through focusing on elements of a therapy that are most likely to be effective for a specific individual patient. Second, it hinders the development of novel treatments which engage specific mechanisms more effectively.

CBT and other psychotherapeutic approaches were in part driven by the rich cognitive and learning theories of the middle of the 20th century. A huge amount of progress has been made since then in cognitive and computational neuroscience leading to a better understanding of the relevant learning and cognitive processes from a basic research point of view. Here, we consider whether these advances have potential to elucidate the functioning of existing interventions, and whether they could support the back-translation into novel psychotherapeutic modules.

\section{Mechanisms and Mediators in Psychotherapy Research}

The study of the mechanisms underlying the efficacy of psychological treatments has focused on the identification of mediators. A mediator is defined as a variable which is on the causal path linking the intervention with the treatment effect ${ }^{6}$. As an example, a popular idea posits that cognitive change, i.e. change in dysfunctional beliefs, accounts for improvement in depressive symptoms brought about by cognitive therapy ${ }^{7}$. In order to formally demonstrate that cognitive change is a mediator of cognitive therapy, a cognitive therapy needs to change the mediating variable ("dysfunctional beliefs") and reduce depressive symptoms. Moreover, the change in the mediator needs to precede, and to be proportional to, the reduction of symptoms. Excellent reviews have noted important limitations of the existing evidence on psychotherapy mechanisms and mediators, including inadequate control conditions, limited sample sizes, and a focus on differences between groups of individuals with and without a diagnosis ${ }^{6,8}$. Here, we describe limitations associated with the measurement and conceptualization of the mechanisms themselves.

First, despite their indisputably high value in clinical practice, there are difficulties around the use of selfreport instruments to measure mechanisms. Consider treatments for anhedonia. Items assessing anhedonia in standardized questionnaires will often ask whether individuals were able to enjoy things they had previously enjoyed. This precise wording may have been used in the therapy session, with therapists encouraging individuals to identify sources of enjoyment, seek them out, and focus on the enjoyment. As such, self-report is likely to be affected by social desirability effects ${ }^{9}$. The repeated application of self-report questionnaires might even exert a psychoeducational effect ${ }^{10}$. Other systematic biases which might impact on the validity of self-reports are known, such as memory or recency effects in depression ${ }^{11,12}$. Furthermore, the measurement of self-reported symptoms and self-reported mediators might be conflated as items in both types of questionnaires can bear similarity. As such, self-report instruments such as questionnaires, and pre-post changes therein might function more as "tests of knowledge" rather than indexing true cognitive or behavioural change ${ }^{13}$.

Second, past mediator studies have tended to examine full therapeutic approaches (for example, comparing CBT, psychodynamic therapy and psychoanalysis ${ }^{14}$ ) and their relation to potential mediators. However, each full psychotherapy involves multiple component interventions (Fig. 1A) and any individual is likely only to benefit from a subset of these interventions. For instance, in Fig. 1B, the interventions specific to Therapy 1 are generally more effective, but some individuals respond better to the interventions in Therapy 2. The effect size when comparing two therapies is linear in the difference between the average response probability to individual components (Fig. 1C), making large effects in the comparison very unlikely. The presence of shared interventions can confound differences, and even have a multiplicative effect on the ability to discover a difference in efficacy (Fig 1C; a version of the 'dodo bird' effect). If 
measurements of mechanisms allowed specific interventions to be delivered only to sensitive individuals, the treatment effects would be improved.

Third, and related to the previous point, mechanistic studies have at times examined mediators at a coarse scale. For example, there is evidence that dysfunctional cognitions measured with the Dysfunctional Attitude Scale (DAS ${ }^{15}$ ) are a mediator of cognitive therapy (see ${ }^{8,13}$ ). However, the DAS consists of various (i.e. 2-4) uncorrelated factors ${ }^{16}$. Of course, this could be addressed by using scales which have better internal consistency and load on a single factor. However, this approach is unlikely to ever work fully: after all, these processes interact in complex manners and do not just superimpose linearly.

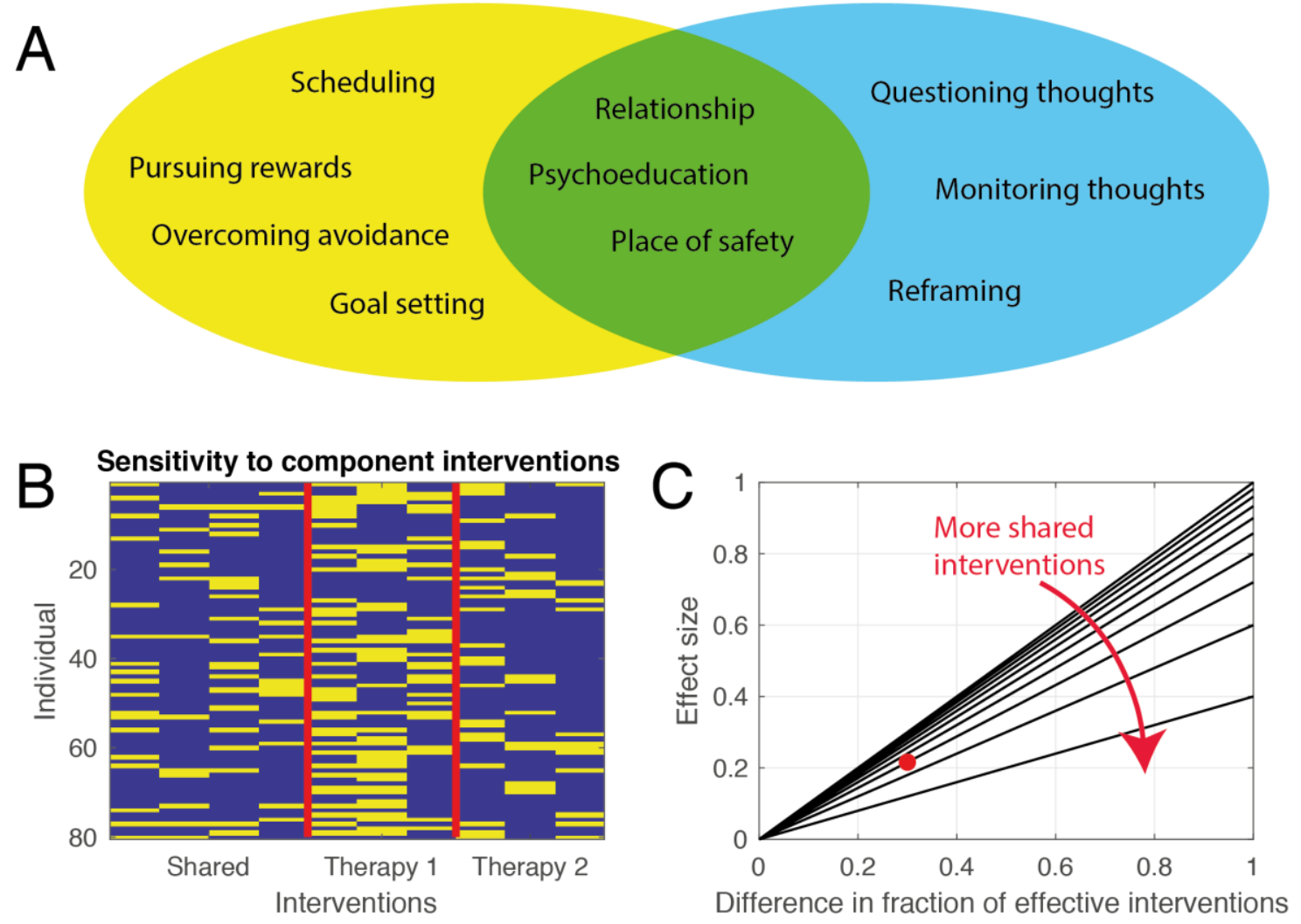

Figure 1: A) Different psychotherapeutic approaches (yellow: Behavioural Activation; blue: Cognitive Restructuring) contain both shared and specific interventions. B) Individuals may respond or be sensitive to only a fraction of both the shared and specific components (yellow), while they may be insensitive and unresponsive to the other interventions. $A$ therapy is better if individuals have a high probability of responding to some of its specific interventions. Here, the probability of responding to the three interventions specific to Therapy 1 is 0.5 , while the probability of responding to either the shared or the Therapy 2 interventions is 0.2 C) Simulations based on an effect size of 1 when comparing therapies to wait-list controls. The effect size for comparing two therapies is linear in the difference in response probability to each intervention, and the fraction of shared interventions can have a multiplicative effect on the ability to observe a difference. The red dots show the example in panel B. A precision psychotherapy approach would allow the delivery of only those interventions to which the individual is sensitive. This would increase the fraction of effective interventions while also reducing shared interventions, and hence overall increase the effect size.

\section{Refining mediators - neuro-computational mechanisms of psychotherapy components}

One potentially useful approach to address these limitations is the integration of experimental paradigms from computational cognitive neuroscience research into psychotherapy research. These paradigms have been developed and validated as quantitative, objective measurements of underlying cognitive processes. Over the last two decades, these paradigms have often times been combined with measurements of brain function using fMRI, EEG or MEG, to dissect the neural correlates of these processes. In some cases, such tasks are derived from translational research, frequently offering even more detailed insight into the neurofunctional and neuro-pharmacological underpinnings of a cognitive process of interest. Specifically, the computational models employed to capture the underlying cognitive processes are inspired by our knowledge about how neurons implement and compute such processes. Hence, we termed them "neurocomputational" mechanisms. When combined with computational modelling, they can disentangle complex interacting latent factors that jointly shape cognition, learning, generalization and affect. Some types of computational models ("generative models") allow for mechanistically interpretable computational parameters to be inferred (e.g. ${ }^{17-21}$, see also ${ }^{22}$ ). There is recent evidence that such neuro-computational 
measures may provide estimates of hidden, disease-relevant processes that can usefully predict treatment trajectories ${ }^{23-25} .{ }^{26}$

Such neuro-computational measures are promising because they address several of the issues identified in the measurement of psychotherapy mechanisms identified above. First, psychotherapeutic interventions often explicitly target the kinds of behaviours and cognitions the computational-cognitive tasks are designed to asses - core point we will elaborate on below. Second, by relying on objective features of behaviour, neuro-computational probes circumvent some of the problems associated with self-reports outlined above: They are not as strongly influenced by the subjective views and, hence, less susceptible to social desirability, nor do they show similarities to symptom ratings.

However, these methods have only rarely been applied to identify mediators of psychotherapy ${ }^{27,28}$, and where this has been done, they have been deployed in pre-post designs examining complete psychotherapy packages. This shares the difficulties of comparing complete psychotherapies outlined above: Even if a neuro-computational measure is found to be altered by a psychotherapy, the presence of shared interventions impedes the assignment of this effect to a particular psychotherapy (Fig. 1). Furthermore, the co-delivery of other interventions with partial efficacy will confound the association.

As a result, there has been minimal research attempting to relate specific interventions to specific underlying mechanisms. Recent advances allow behavioural task measures to be easily deployed online in repeated-measures longitudinal designs ${ }^{29}$. This allows for novel study designs where changes in specific underlying mechanisms are associated with specific interventions.

\section{Back-translating innovations of Cognitive Neuroscience into Psychotherapy mediator research}

To illustrate the approach, we discuss two candidate neuro-computational probes involving confidence and reward-effort trade-offs in the treatment of depression. 


\section{A}

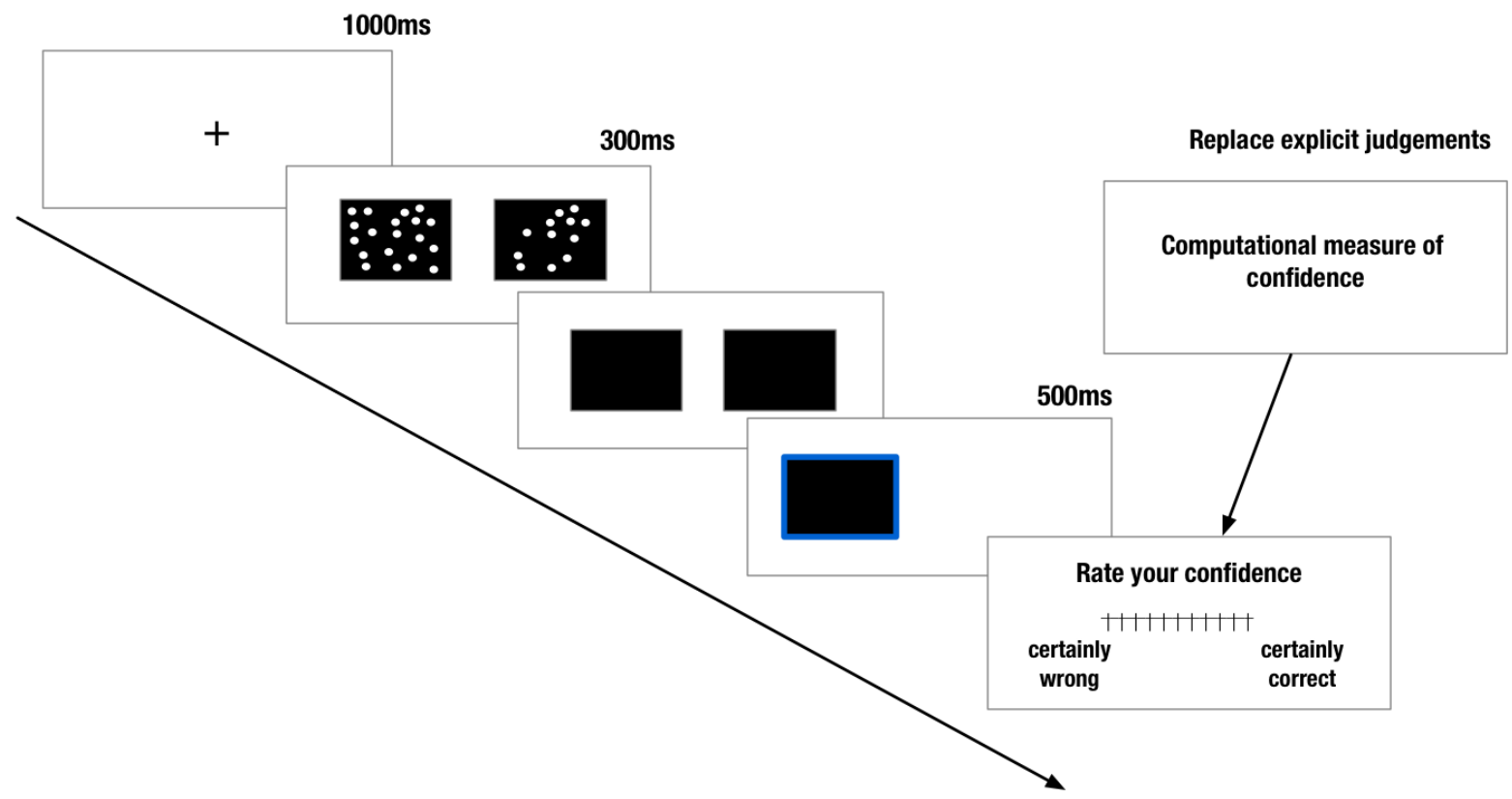

B

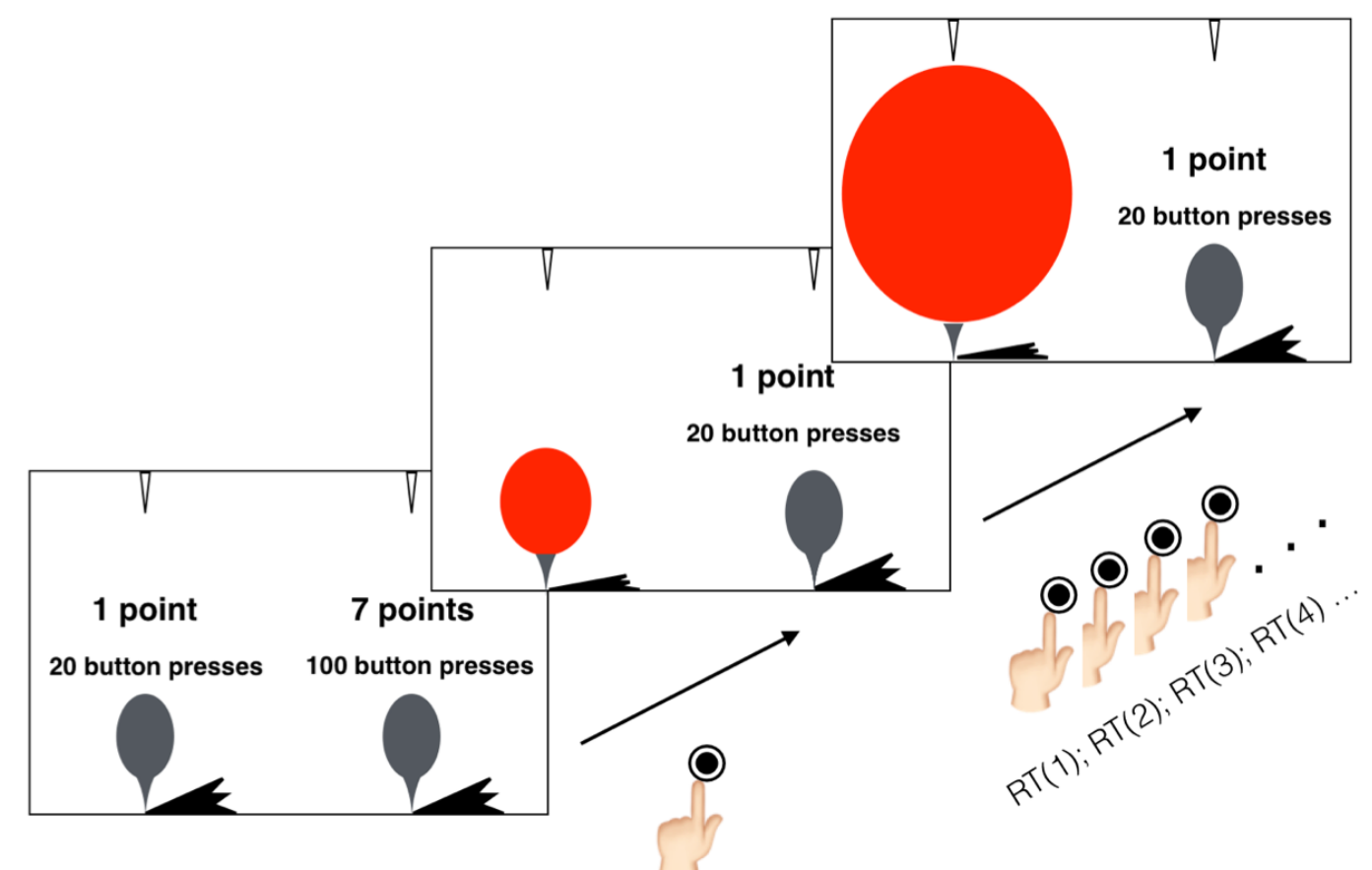

Figure 2. A) A simple perceptual decision-making task where participants are asked to judge which box contains a larger number of dots. Traditionally, to measure confidence, participants are asked to retrospectively give their confidence ratings. We suggest a framework for measuring individual confidence profiles via a computational measure that has been shown to robustly infer confidence from objective performance data (i.e. how long it takes subjects to respond, and how accurate they are). Note that the perceptual task can be replaced with a more engaging task, e.g. a memory task where subjects are asked to memorize a set of objects. B) Physical effort for reward task. On each trial participants need to decide between investing more effort (e.g. 100 button presses) for a higher reward (e.g. 7 points) or less effort (here 20 button presses) for a smaller reward (here 1 point). They need to indicate their choice with a button press. The choices relate to the anticipation of effort ( $\left.E_{\text {ant }}\right)$ and reward $\left(R_{a n t}\right)$. These quantities can be estimated by means of a computational model. The chosen effort needs to be executed by means of button presses. The reaction times $(R T)$ between those button presses can be used to compute the vigour, which relates to the experience of the effort $\left(E_{\text {exp }}\right)$ during the trial. 


\section{Confidence as a candidate mediator for cognitive therapy in depression}

Patients with depression often show biases towards low confidence when judging their own accomplishments and abilities. "Feelings of worthlessness" or "inappropriate guilt" are amongst the DSM$V$ criteria for depression and under-confidence when performing simple perceptual decision-making tasks is associated with depressive symptoms ${ }^{18}$ and lower self-esteem ${ }^{30}$. Negative self-evaluation is also a key element of a prominent cognitive theory of depression ${ }^{31}$. CBT targets negative self-evaluation through Cognitive Restructuring (CR) ${ }^{7}$. This involves first identifying patients' negative thought patterns and pessimistic assumptions about themselves, which includes the general feeling of low confidence in their abilities (e.g., "I am performing worse at work than my colleagues", "I am not an interesting conversation partner"). CR trains individuals to notice the automatic thought patterns in day-to-day life, to question and deconstruct them ("What evidence is there that this thought isn't accurate?") and to replace them with more realistic thought patterns.

CR can be viewed as training of metacognitive bias and confidence. In cognitive neuroscience research, confidence and metacognitive bias have been extensively studied in tasks via repeated retrospective confidence judgements after making a decision (Fig. 2A). Computational models can directly infer confidence levels from objective performance data, e.g. choices and response times ${ }^{32}$. Such computational measures of metacognition enable the implicit measurement of confidence. A recent theoretical framework 33,34 posits a two-way relationship between metacognitive ability in a domain and broader beliefs about self-ability. It suggests that confidence levels measured in specific domains, e.g. through tasks, could be a proxy for measures such as self-efficacy, or broader confidence judgements. Hence, the possibility that CR might influence negative self-evaluation by altering metacognitive processes can be tested by examining whether task-level metacognition mediates the impact of $\mathrm{CR}$ on self-evaluation. Metacognition as a mediator would thereby provide a link between symptoms of (and interventions to treat) depression and the neurobiology of metacognition. Importantly, the use of the task as a measure of the metacognitive mechanism might address some of the issues raised above.

\section{Reward- and Effort-based Decision-Making as Candidate Mediators for Behavioural Activation}

Patients with depression also show reduced engagement in rewarding activities ${ }^{35}$. The decision to engage in rewarding activities (e.g. going out, meeting friends) compared to "depressive" behaviours (staying in bed) can be viewed as a trade-off between the anticipated reward and the anticipated effort for each behaviour ${ }^{23}$. The reduction in rewarding activities seen in depression might hence result from decreased anticipated reward or from increased anticipated effort. Behavioural Activation (BA), a widely disseminated first-line therapy for depression ${ }^{36,37}$ contains component interventions that aim to directly address these aspects: planning and the scheduling of rewarding activities. The aim of planning is to ensure activities are realistic and achievable, thereby reducing the probability that effort will be spent without achieving a goal. The aim of scheduling rewarding activities is to ensure rewards are experienced. The underlying assumption is that the experience of successful planning and rewarding activities re-establish reward and effort expectations. Computational accounts of learning from reinforcement ${ }^{38}$ suggest that learning, i.e. the impact of the outcomes, is driven by the differential between the actually received reinforcement and the expectation, e.g. for the rewards:

$$
R_{\text {ant }}(t+1)=R_{\text {ant }}(t)+\alpha *\left(R_{\text {exp }}(t)+-R_{\text {ant }}(t)\right)
$$

where $\mathrm{t}$ indexes a specific situation, $R_{\text {ant }}(t+1)$ is the anticipated reward which will influence the decision to engage in an activity in the next situation, $R_{\text {exp }}(t)$ is the experience of reward during execution of the planned activity and $\alpha$ is a learning rate. Thus, a change in $R_{\text {ant }}$ needs to occur for patients to engage more in an activity. Hence, $R_{\text {ant }}$ might be a mediator of the effect of BA on the depressive symptom pattern. The model also indicates that for $R_{\text {ant }}$ to be increased $R_{\text {exp }}$ needs to be larger than $R_{\text {ant }}$ and the learning rate $\alpha$ needs to be larger than 0 . Hence, both, the capacity to learn and the capacity to experience reward might be perquisites for BA to work and moderators of its impact. The anticipation of effort can be updated according to the same update rule as the anticipation of reward.

There is a large literature on learning from rewards, and on the trade-off between rewards and efforts ${ }^{39-42}$. In one simple task, different amounts of effort yield different rewards ${ }^{23,43}$ (Fig. 2B). A generative computational model of behaviour in this task includes parameters indexing effort and reward sensitivity (implemented as a trade-off between the necessary effort and the resulting reward anticipation), the vigour used to execute the effortful behaviour and the reaction to the reward (experience). It also formalizes the influence of previous effortful behaviour and reward exposure on new choices as prediction errors 17,23,44. 
Such probes should hence have value as measurements of the putative mechanisms underlying the component interventions of BA.

\section{Limitations}

However, the use of neuro-computational measures in psychotherapy mediator research also faces several substantial challenges. Most importantly, cognitive tasks were recently found to be poor predictors of selfreport measures, and of self-reported real-world outcomes in a study evaluating predictive power in the domain of self-control ${ }^{45}$. Given the importance of self-reported symptom change in motivating treatment seeking and efficacy, this is an important challenge. One potentially addressable reason might be poor psychometric properties of commonly-used tasks, including poor test-retest validity ${ }^{46},{ }^{47}$. These could potentially be addressed by enhancing the number of trials ${ }^{48}$, by combining different behavioural read-outs (e.g. choices, reaction times) in a computational model ${ }^{49}$, or by optimizing model estimation approaches 50,51 . Recent approaches have also combined self-report of subjective well-being or pain with objective measures (task behaviour, fMRI ${ }^{52,53}$ ).

\section{Conclusion}

Neuro-computational measurements are promising mediators of specific psychotherapeutic interventions. They are objective and capture actual behaviour rather than subjective thoughts about behaviour, which is both a strength and a weakness. They profit from a rich neuroscientific and computational underpinning, relating them to normative models of brain function and often allow for detailed quantitative studies of the underlying neural mechanisms. Being often derived from translational research they allow for precise hypotheses regarding the associations of cognitive processes with certain neurotransmitter systems - an important aspect when it comes to tailoring combined (pharmaco-/psychological) treatment approaches. Nevertheless, substantial obstacles exist not only for using tasks in psychotherapy research, but also more broadly for measuring inter-individual differences in general ${ }^{54}$. Research should urgently focus on addressing these obstacles in order to allow for their potential as mediators of psychological interventions to be tested.

\section{References}

1 Patel, V. et al. Addressing the burden of mental, neurological, and substance use disorders: key messages from Disease Control Priorities. The Lancet 387, 1672-1685 (2016).

2 Saxena, S., Thornicroft, G., Knapp, M. \& Whiteford, H. Resources for mental health: scarcity, inequity, and inefficiency. The lancet 370, 878-889 (2007).

3 Davidson, J. R. Major depressive disorder treatment guidelines in America and Europe. The Journal of clinical psychiatry 71, 4-4 (2010).

$4 \quad$ Cuijpers, P. et al. The efficacy of psychotherapy and pharmacotherapy in treating depressive and anxiety disorders: A meta-analysis of direct comparisons. World Psychiatry 12, 137-148 (2013).

5 Luborsky, L. et al. The dodo bird verdict is alive and well-mostly. Clinical Psychology: Science and Practice 9, 2-12 (2002).

6 Kazdin, A. E. Mediators and mechanisms of change in psychotherapy research. Annu. Rev. Clin. Psychol. 3, 1-27 (2007).

7 Beck, A. T. Cognitive therapy of depression. (Guilford press, 1979).

8 Lemmens, L. H., Müller, V. N., Arntz, A. \& Huibers, M. J. Mechanisms of change in psychotherapy for depression: an empirical update and evaluation of research aimed at identifying psychological mediators. Clinical Psychology Review 50, 95-107 (2016).

9 Fastame, M. C. \& Penna, M. P. Does social desirability confound the assessment of self-reported measures of well-being and metacognitive efficiency in young and older adults? Clinical Gerontologist 35, 239-256 (2012).

10 Cuijpers, P., Muñoz, R. F., Clarke, G. N. \& Lewinsohn, P. M. Psychoeducational treatment and prevention of depression: the "Coping with Depression" course thirty years later. Clinical psychology review 29, 449-458 (2009).

11 Paulus, M. P. \& Stein, M. B. Interoception in anxiety and depression. Brain structure and Function 214, 451-463 (2010).

12 Ben-Zeev, D., Young, M. A. \& Madsen, J. W. Retrospective recall of affect in clinically depressed individuals and controls. Cognition and Emotion 23, 1021-1040 (2009). 
Cristea, I. A. et al. The effects of cognitive behavior therapy for adult depression on dysfunctional thinking: A meta-analysis. Clinical Psychology Review 42, 62-71 (2015).

14 Klug, G., Henrich, G., Filipiak, B. \& Huber, D. Trajectories and mediators of change in psychoanalytic, psychodynamic, and cognitive behavioral therapy. Journal of the American Psychoanalytic Association 60, 598-605 (2012).

15 Weissman, A. N. \& Beck, A. T. Development and validation of the Dysfunctional Attitude Scale: A preliminary investigation. (1978).

16 De Graaf, L. E., Roelofs, J. \& Huibers, M. J. Measuring dysfunctional attitudes in the general population: The Dysfunctional Attitude Scale (form A) Revised. Cognitive therapy and research 33, 345 (2009).

17 Huys, Q. J., Pizzagalli, D. A., Bogdan, R. \& Dayan, P. Mapping anhedonia onto reinforcement learning: a behavioural meta-analysis. Biology of mood \& anxiety disorders 3, 12 (2013).

18 Rouault, M., Seow, T., Gillan, C. M. \& Fleming, S. M. Psychiatric symptom dimensions are associated with dissociable shifts in metacognition but not task performance. Biological psychiatry 84, 443-451 (2018).

19 Gillan, C. M., Kosinski, M., Whelan, R., Phelps, E. A. \& Daw, N. D. Characterizing a psychiatric symptom dimension related to deficits in goal-directed control. elife 5, e11305 (2016).

Patzelt, E. H., Kool, W., Millner, A. J. \& Gershman, S. J. Incentives boost model-based control across a range of severity on several psychiatric constructs. Biological psychiatry 85, 425-433 (2019).

21 Lieder, F., Chen, O. X., Krueger, P. M. \& Griffiths, T. L. Cognitive prostheses for goal achievement. Nature human behaviour 3, 1096-1106 (2019).

22 Huys, Q. J., Browning, M., Paulus, M. \& Frank, M. J. Advances in the computational understanding of mental illness. Neuropsychopharmacology, 1-19 (2020).

23 Berwian, I. M. et al. Computational mechanisms of effort and reward decisions in patients with depression and their association with relapse after antidepressant discontinuation. JAMA psychiatry (2020).

24 Konova, A. B. et al. Computational Markers of Risky Decision-making for Identification of Temporal Windows of Vulnerability to Opioid Use in a Real-world Clinical Setting. JAMA psychiatry (2019).

25 Harle, K. M. et al. Bayesian neural adjustment of inhibitory control predicts emergence of problem stimulant use. Brain 138, 3413-3426 (2015).

26 Marwood, L., Wise, T., Perkins, A. M. \& Cleare, A. J. Meta-analyses of the neural mechanisms and predictors of response to psychotherapy in depression and anxiety. Neuroscience \& Biobehavioral Reviews 95, 61-72 (2018).

27 Queirazza, F., Fouragnan, E., Steele, J. D., Cavanagh, J. \& Philiastides, M. G. Neural correlates of weighted reward prediction error during reinforcement learning classify response to cognitive behavioral therapy in depression. Science advances 5, eaav4962 (2019).

28 Wheaton, M. G., Gillan, C. M. \& Simpson, H. B. Does cognitive-behavioral therapy affect goaldirected planning in obsessive-compulsive disorder? Psychiatry Research 273, 94-99 (2019).

29 Rutledge, R. B., Chekroud, A. M. \& Huys, Q. J. Machine learning and big data in psychiatry: toward clinical applications. Current opinion in neurobiology 55, 152-159 (2019).

30 Moses-Payne, M. E., Rollwage, M., Fleming, S. M. \& Roiser, J. P. Post-decision evidence integration and depressive symptoms. Frontiers in psychiatry 10, 639 (2019).

31 Beck, A. T. Depression: Clinical, experimental, and theoretical aspects. (Hoeber Medical Division, Harper \& Row, 1967).

32 Atiya, N. A. A., Huys, Q., Dolan, R. J. \& Fleming, S. M. Explaining distortions in metacognition with an attractor network model of decision uncertainty. bioRxiv, 2020.2009.2025.313619, doi:10.1101/2020.09.25.313619 (2020).

33 Rouault, M., McWilliams, A., Allen, M. G. \& Fleming, S. M. Human metacognition across domains: insights from individual differences and neuroimaging. Personality neuroscience 1 (2018). 
34 Mazancieux, A., Fleming, S. M., Souchay, C. \& Moulin, C. J. Is there a G factor for metacognition? Correlations in retrospective metacognitive sensitivity across tasks. Journal of Experimental Psychology: General (2020).

35 Association, A. P. Diagnostic and Statistical Manual of Mental Disorders (DSM-5 ${ }^{\circledR}$ ). (American Psychiatric Pub, 2013).

36 Lewinsohn, P. M. A behavioral approach to depression. Essential papers on depression, 150-172 (1974).

37 Nagy, G. A. et al. Reward network modulation as a mechanism of change in behavioral activation. Behavior modification 44, 186-213 (2020).

38 Schultz, W., Dayan, P. \& Montague, P. R. A neural substrate of prediction and reward. Science 275, 1593-1599 (1997).

39 Daw, N. D. \& Dayan, P. The algorithmic anatomy of model-based evaluation. Philos Trans R Soc Lond B Biol Sci 369, doi:10.1098/rstb.2013.0478 (2014).

40 Keren, H. et al. Reward processing in depression: a conceptual and meta-analytic review across fMRI and EEG studies. American Journal of Psychiatry 175, 1111-1120 (2018).

41 Dolan, R. J. \& Dayan, P. Goals and habits in the brain. Neuron 80, 312-325, doi:S08966273(13)00805-2 [pii]

10.1016/j.neuron.2013.09.007 (2013).

42 Treadway, M. T., Bossaller, N. A., Shelton, R. C. \& Zald, D. H. Effort-based decision-making in major depressive disorder: a translational model of motivational anhedonia. Journal of abnormal psychology 121, 553 (2012).

43 Gold, J. M. et al. Negative symptoms of schizophrenia are associated with abnormal effort-cost computations. Biol Psychiatry 74, 130-136, doi:S0006-3223(13)00035-8 [pii]

10.1016/j.biopsych.2012.12.022 (2013).

44 Niv, Y., Daw, N. D., Joel, D. \& Dayan, P. Tonic dopamine: opportunity costs and the control of response vigor. Psychopharmacology 191, 507-520 (2007).

45 Eisenberg, I. W. et al. Uncovering the structure of self-regulation through data-driven ontology discovery. Nature communications 10, 1-13 (2019).

46 Hedge, C., Powell, G. \& Sumner, P. The reliability paradox: Why robust cognitive tasks do not produce reliable individual differences. Behavior Research Methods 50, 1166-1186 (2018).

47 Enkavi, A. Z. et al. Large-scale analysis of test-retest reliabilities of self-regulation measures. Proceedings of the National Academy of Sciences 116, 5472-5477 (2019).

48 Rouder, J. N. \& Haaf, J. M. A psychometrics of individual differences in experimental tasks. Psychonomic bulletin \& review 26, $452-467$ (2019).

49 Shahar, N. et al. Improving the reliability of model-based decision-making estimates in the twostage decision task with reaction-times and drift-diffusion modeling. PLoS computational biology 15, e1006803 (2019).

50 Brown, V. M., Chen, J., Gillan, C. M. \& Price, R. B. Improving the reliability of computational analyses: Model-based planning and its relationship with compulsivity. Biological Psychiatry: Cognitive Neuroscience and Neuroimaging (2020).

51 Haines, N. et al. Learning from the Reliability Paradox: How Theoretically Informed Generative Models Can Advance the Social, Behavioral, and Brain Sciences. (2020).

52 Rutledge, R. B. et al. Association of neural and emotional impacts of reward prediction errors with major depression. JAMA psychiatry 74, 790-797 (2017).

53 Wager, T. D. et al. An fMRI-based neurologic signature of physical pain. New England Journal of Medicine 368, 1388-1397 (2013).

54 Flake, J. K. \& Fried, E. I. Measurement schmeasurement: Questionable measurement practices and how to avoid them. (2019). 
Queirazza, F., Fouragnan, E., Steele, J. D., Cavanagh, J. \& Philiastides, M. G. Neural correlates of weighted reward prediction error during reinforcement learning classify response to cognitive behavioral therapy in depression. Science advances 5, eaav4962 (2019). **

This study is an example for using task-based fMRI in combination with computational modelling to predict treatment response in CBT. Combining computational modelling of a behaviour with modern multivariate brain analysis methods, the authors found that psychotherapy responders exhibit greater pre-treatment neural activity associated with a weighted reward prediction error in the right striatum and right amygdala. Based on this signal, significant out-of-sample classification of treatment response was achieved. Even though the authors did not perform a formal mediation analysis, building on their repeated task-based fMRI assessment, they could demonstrate that change in symptoms was correlated with change in prediction error related activation in the amygdala. I

Berwian, I. M. et al. Computational mechanisms of effort and reward decisions in patients with depression and their association with relapse after antidepressant discontinuation. JAMA psychiatry (2020). *

This paper is an example of using computational modelling to tackle a specific clinical problem, namely relapse prediction after antidepressant discontinuation. Applying a computational model to behavioral data collected by means of a physical effort task, the author showed that patients invested less effort for reward than controls due to increased effort sensitivity. Patients who later relapsed after antidepressant discontinuation took longer to decide between high and low effort/reward options, which could be explained by a higher boundary in a drift-diffusion model in these patients. These higher decision times predicted relapse better than chance in a validation sample.

Konova, A. B. et al. Computational Markers of Risky Decision-making for Identification of Temporal Windows of Vulnerability to Opioid Use in a Real-world Clinical Setting. JAMA psychiatry (2019).*

This paper exemplifies the use of repeated task measurements to derive neuro-cognitive probes to describe clinical trajectories. The authors applied a simole risk taking task in a sample of treatment seeking opioid abusers. The results showed that a computationally derived parameterm, namely ambiguity tolerance, was significantly associated with increased odds of prospective opioid use. The authors conclude that patients were more tolerant specifically of ambiguous risks prior to these use events.

Rouault, M., Seow, T., Gillan, C. M. \& Fleming, S. M. Psychiatric symptom dimensions are associated with dissociable shifts in metacognition but not task performance. Biological psychiatry 84, 443451 (2018).*

This paper tackled the association of metacognition with psychiatric symptoms by using an online version of a simple perceptual metacognition task in a large general population sample. The authors could demonstrate that anxious-depressive symptoms were associated with lower confidence and heightened metacognitive efficiency, whereas compulsivity was associated with higher confidence and lower metacognitive efficiency.

Eisenberg, I. W. et al. Uncovering the structure of self-regulation through data-driven ontology discovery. Nature communications 10, 1-13 (2019). **

This paper is an important contribution investigating construct validity when measuring self-regulation. The authors report on a fairly large online assessment of widely used tasks as well as questionnaires assessing self-regulation. They could demonstrate that questionnaires and task which both are thought to putatively measure self-regulation show little empirical correlation. Problematically indeed for the use of tasks in clinical practice, but also for inter-individual difference research in general, is that tasks showed little predictive power for real-life self-control.

Flake, J. K. \& Fried, E. I. Measurement schmeasurement: Questionable measurement practices and how to avoid them. (2019). ${ }^{*}$ (accepted in Advances in Methods and Practices in Psychological Science)

Based on the example of measuring depression, this paper offers a thoughtful overview over common problems with measurement in psychological science and raises awareness on how they diminish the validity of study conclusions. The authors offer suggestions on how to circumvent measurement problems. 


\section{Conflict of Interest}

The authors report no conflict of interest 